\title{
Effects of Aroma Massage on Home Blood Pressure, Ambulatory Blood Pressure, and Sleep Quality in Middle-Aged Women with Hypertension
}

\author{
Myeong-Sook Ju, ${ }^{1}$ Sahng Lee, ${ }^{2}$ Ikyul Bae, ${ }^{3}$ Myung-Haeng Hur, \\ Kayeon Seong, ${ }^{4}$ and Myeong Soo Lee ${ }^{5}$ \\ ${ }^{1}$ Eulji University Hospital, Daejeon 302-799, Republic of Korea \\ ${ }^{2}$ Smart Hospital, Daejeon 302-859, Republic of Korea \\ ${ }^{3}$ Kunsan College of Nursing, Gunsan 573-719, Republic of Korea \\ ${ }^{4}$ College of Nursing, Eulji University, 143-5 Yongdudong, Jung-gu, Daejeon 302-832, Republic of Korea \\ ${ }^{5}$ Medical Research Division, Korea Institute of Oriental Medicine, Daejeon 305-811, Republic of Korea
}

Correspondence should be addressed to Myung-Haeng Hur; mhhur@eulji.ac.kr

Received 22 October 2012; Revised 8 December 2012; Accepted 9 December 2012

Academic Editor: Jenny M. Wilkinson

Copyright (C) 2013 Myeong-Sook Ju et al. This is an open access article distributed under the Creative Commons Attribution License, which permits unrestricted use, distribution, and reproduction in any medium, provided the original work is properly cited.

\begin{abstract}
The purpose of this study was to evaluate the effects of aroma massage applied to middle-aged women with hypertension. The research study had a nonequivalent control group, nonsynchronized design to investigate the effect on home blood pressure (BP), ambulatory BP, and sleep. The hypertensive patients were allocated into the aroma massage group $(n=28)$, the placebo group $(n=28)$, and the no-treatment control group $(n=27)$. To evaluate the effects of aroma massage, the experimental group received a massage with essential oils prescribed by an aromatherapist once a week and body cream once a day. The placebo group received a massage using artificial fragrance oil once a week and body cream once a day. BP, pulse rate, sleep conditions, and 24-hour ambulatory $\mathrm{BP}$ were monitored before and after the experiment. There was a significant difference in home systolic blood pressure $(\mathrm{SBP})(F=6.71, P=0.002)$ between groups after intervention. There was also a significant difference in SBP $(F=13.34, P=0.001)$ and diastolic blood pressure (DBP) $(F=8.46, P=0.005)$ in the laboratory between aroma massage and placebo groups. In sleep quality, there was a significant difference between groups $(F=6.75, P=0.002)$. In conclusion, aroma massage may help improve patient quality of life and maintain health as a nursing intervention in daily life.
\end{abstract}

\section{Introduction}

A chronic disease refers to a disease state progressing over a long period of time, and chronic diseases are responsible for $63 \%$ of overall deaths worldwide [1]. Of the chronic diseases in South Korea, the prevalence of hypertension in people over the age of 30 increased from $24.6 \%$ in 2007 to $26.9 \%$ in 2008 [2]. In particular, women show an increasing prevalence with age $(4.3 \%$ of women in their $30 \mathrm{~s}, 15.0 \%$ in their $40 \mathrm{~s}, 32.6 \%$ in their $50 \mathrm{~s}$, and $50.2 \%$ in their $60 \mathrm{~s}$ ) to the extent that half of the women over the age of 60 had hypertension [2].

The first action for BP control is BP measurement. Because the results of $\mathrm{BP}$ measurement are influenced by multiple factors including time of day, location, and activity level, the accurate measurement and interpretation of $\mathrm{BP}$ are critical to diagnose and track hypertension. Accordingly, home BP measurements by the patient and 24-hour ambulatory $\mathrm{BP}$ measurements in addition to simple office sitting $\mathrm{BP}$ measurements are meaningful [3]. The measurement of 24-hour ambulatory BP in hypertensive patients aims to identify the degree of nocturnal decrease in BP, leading to the increased interest in quality of sleep, sleep disorders, and duration of sleep. In general, night-time BP decreases due to increased parasympathetic nervous system activity and decreased sympathetic nervous system activity during sleep [3]. Thus, the presence of sleep disorders, the duration of 
sleep, and the quality of sleep can influence hypertension [4].

The management of hypertension aims at preventing end organ complications and mortality due to hypertension, with the target $\mathrm{BP}$ below $140 / 90 \mathrm{mmHg}[3,5]$. The goal of nursing intervention is to increase patient understanding of the hypertension process and its treatment and increase patient involvement in self-management programs to ultimately prevent hypertension complications [5].

Recently, there has been an increasing interest in holistic interventions combining traditional and complementary therapies for health promotion and maintenance. Aromatherapy is a form of complementary therapy using essential oil extracted from plants. The oil is applied through different means to achieve various effects through its chemical properties and application methods [6, 7]. Of the aromatherapy essential oils, lavender (Lavandula officinalis), marjoram (Origanum majorana), ylang-ylang (Cananga odorata), and Neroli (Citrus aurantium) are used for hypertensive patients. Lavender is the selective oil that balances the nervous system and alleviates insomnia, while marjoram activates parasympathetic nervous system and relaxes the sympathetic nervous system. Ylang-ylang controls cardiac palpitations and hypertension, and Neroli oil is effective in insomnia and depression. The main chemical ingredients of lavender, marjoram, ylangylang, and neroli are linalyl acetate, terpinen-4-ol, benzyl acetate, and limonene, respectively [6].

Aroma massage combines massage with essential oils to stimulate blood and lymphatic circulation and improve oxygen and nutrient supply, and it is effective for the relaxation of tense muscles, pain relief, and alleviation of emotional disturbances $[6,8,9]$. Women in middle age experience changes in the autonomic nervous system such as increases in adrenal cortex hormones and catecholamines, leading to increased $\mathrm{BP}$ and unsuccessful psychological management, which can have effects such as depression, anxiety, and insomnia $[10,11]$. Recent research on the effects of aromatherapy on BP and sleep has targeted menopausal women [12], prehypertensive middle-aged women [13], and stage 1 hypertensive patients [14]. But there have been no studies using aroma massage and body cream as a method of aromatherapy.

Thus, the aim of this study was to evaluate the effect of aroma massage on home $\mathrm{BP}$, office $\mathrm{BP}$, ambulatory $\mathrm{BP}$, and sleep quality.

\section{Methods}

2.1. Study Design. This study is a nonequivalent control group, nonsynchronized study intended to compare the effects of aroma massage on home BP, office BP, ambulatory $\mathrm{BP}$, and sleep in middle-aged women with hypertension (Figure 1). To prevent the spread and contamination of data as a part of the nonsynchronized study design, the control group data were collected before the data from the intervention group (aroma massage group) and the placebo group (artificial fragrance massage group).

2.2. Participants and Data Collection. The participants were recruited between March 15 and June 30 of 2011 at E

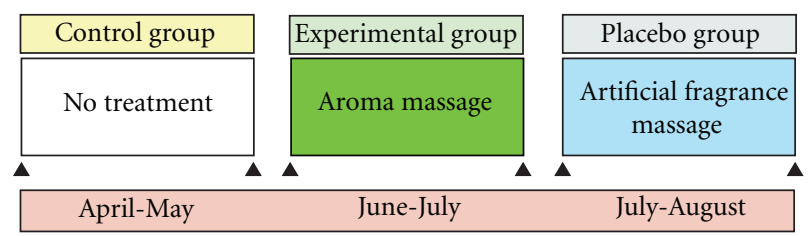

Figure 1: Study design.

University Hospital in D city, outpatient clinic in the department of internal medicine. Ethical approval was obtained from the institutional review board (IRB) after submission of the research plan. A notice was sent to the department, and the participants were recruited from patients diagnosed with hypertension and receiving ongoing outpatient cardiology followup after consultation with an internal medicine specialist. The eligibility criteria included the following: (1) a diagnosis of hypertension and ongoing followup treatment or consultation at outpatient cardiology clinic; (2) women between the ages of 40 and 59; (3) ability to communicate and describe symptoms; (4) not currently taking anxiolytics or hypnotics; (5) no presence of psychiatric disorders; and (6) providing informed consent regarding the experiment.

The participants were recruited from hypertensive patients using a notice sent to the Internal Medicine Outpatient Clinic; their gender, medications, and conformity to the eligibility criteria were reviewed. The study interventions, measurement methods, and the ability to enroll and drop out of the study were explained to participants, and consent was obtained from those in agreement. The participants were instructed to visit the research office on the intervention day according to the schedule and avoid excessive exercise and diet during the study period and to notify the researchers when there was a change in antihypertensive medication prescription.

2.3. Sample Size Calculation. The sample size of this study was determined using the $\mathrm{G}^{*}$ power program and by assigning alpha value, power, and effect size. The effect size was determined based on a prior aromatherapy study [14] and assigning mean, sample size, and pooled variance in the $G^{*}$ power program. To calculate the sample size, we substituted $\alpha(0.05), 1-\beta(0.80)$, group (3), and effect size (0.34). The sample size was determined to be a total of 90 participants in the three groups using $\mathrm{G}^{*}$ power. In addition, estimating a dropout rate of $10 \%, 33$ participants were assigned to the experimental group, the control group, and the placebo group, for a total of 99 participants.

2.4. Interventions. The intervention used in the experimental group was the application of aroma massage and aroma body cream. The placebo group received massage and body cream with an artificial fragrance, and the control group did not receive any intervention. According to the schedule, there were a total of five sessions in the research office for four weeks. The aroma massage oil used contained essential oils prescribed by an international aromatherapist prepared by blending lavender, marjoram, ylang-ylang, and Neroli at 
a ratio of $20: 10: 15: 2$ followed by dilution to $3 \%$ with a carrier oil prepared by blending almond oil and jojoba oil at ratio of $9: 1$. Such prepared massage oil was preserved at room temperature for use. The aroma body cream was synthesized according to the prescription of an international aromatherapist. The oil base was prepared by combining $100 \mathrm{~mL}$ of jojoba oil, $100 \mathrm{~mL}$ of sweet almond oil, $100 \mathrm{~mL}$ of evening primrose oil, and $30 \mathrm{~mL}$ of olive wax. The water base was prepared by adding $700 \mathrm{~mL}$ of rose water and $20 \mathrm{~g}$ of vitamin $\mathrm{E}$ in a glass beaker and heating in boiling water until the temperature reached over $70 \sim 75^{\circ} \mathrm{C}$, at which point the water base was added to the oil base. The water base combined with oil base was mixed using a blender for 10-15 minutes until the temperature reached below $40^{\circ} \mathrm{C}$, at which point the blending oil used for massage (lavender, marjoram, ylangylang, and Neroli blended in $20: 10: 15: 2$ ratio) was added to produce a $3 \%$ diluted body cream.

Four research assistants providing massage were taught aroma massage theory and massage protocols ( 4 hours) and received practical training (4 hours) from an international aromatherapist. The assistants performed practice massages before providing massages to participants according to the massage protocols. Aroma massage was provided in a research office that was divided into a waiting room and a massage room for study purposes at E University. According to the schedule, once the study participants arrived at the office, they changed into a massage gown and relaxed in a supine position on the bed for 10 minutes followed by BP measurement using a digital sphygmomanometer (Omron, HEM-780, Japan) as a preintervention measurement and massage according to the massage protocol. The massage was performed in the order of back, posterior legs, anterior legs, abdomen, arms, and shoulders. The total amount of oil needed for the massage was approximately $30 \mathrm{~mL}$, and the duration of massage was approximately 1 hour. Ten minutes after the massage was completed, another BP measurement was taken as a postinterventional BP using the same method as the preinterventional measurement, and the participants were provided with $150 \mathrm{~mL}$ of lukewarm water to drink. A total of $200 \mathrm{~mL}$ of prepared aroma body cream was provided to the participants after the first massage, with instructions to apply approximately $10 \mathrm{~mL}$ on arm, legs, and abdomen, excluding the chest and back, after showering and before sleep every day.

Artificial fragrance massage oil and body cream were prepared and used for the placebo group. Almond oil and jojoba oil were blended in a $9: 1$ ratio to produce the carrier oil, in which artificial fragrance was diluted to $3 \%$. The massage oil was stored at room temperature for use. The artificial fragrance body cream was produced and applied by the researchers and research assistants as the same method of making a massage oil and body cream. The control group received no intervention.

\subsection{Outcome Measures}

2.5.1. Blood Pressure. Home BP was measured by participants every Tuesday and Friday at $10 \mathrm{AM}$ after 10 minutes of rest using a home digital BP machine. Two measurements were performed on the left upper arm, and the average of the BP measurements was calculated. The 24-hour ambulatory BP was measured using a 24-hour ambulatory BP monitor (AND TM-2430, Japan). Daytime BP was measured every 30 minutes between $6 \mathrm{AM}$ and 9:30 PM, while the nighttime BP was measured every hour between $10 \mathrm{PM}$ and $6 \mathrm{AM}$ the next morning. These measurements were performed once before and once after the intervention.

2.5.2. Sleep Quality. To assess sleep quality in this study, a translated version of Verran and Synder-Halpern (VSH) Sleep Scale (1987) by Kang (1992) was used. The tool assesses four categories related to beginning of sleep and depth of sleep for a total of 8 questions each on a scale of 0-10 (a total range of $0-80$ ), with higher score corresponding to a higher sleep satisfaction.

2.6. Data Collection and Analyses. Prior to beginning the study, the participants were asked to relax for 10 minutes before measuring their preinterventional BP and obtaining sleep status and 24-hour ambulatory BP measurements. To test the effects of aromatherapy essential oil massage, the experimental group received massage once a week using diluted blended essential oil and daily application of body cream, while the placebo group received a massage with carrier oil with artificial fragrance once a week and daily application of body cream. The control group received no interventions. To measure the postinterventional effect, twice-weekly home BP was measured until week 3; in the fourth week, home BP, 24-hour ambulatory BP, and sleep status were evaluated.

The statistical analysis of the data was performed using SPSS 19.0 software. The general characteristics of participants were analyzed in terms of frequency, number, and percentage using $\chi^{2}$-test and ANOVA. The test for homogeneity on preinterventional dependent variables between the experimental group, the placebo group, and the control group was conducted using ANOVA. The pre- and postinterventional $\mathrm{BP}$ and the sleep status of the participants in the experimental, placebo, and control groups were analyzed using ANOVA, repeated measures ANCOVA, and $\chi^{2}$-test, while the posthoc analysis was performed with the Tukey method. The reliability of the measurement tool for quality of sleep was analyzed using internal reliability Cronbach's $\alpha$ value.

\section{Results}

3.1. Participants. A total of 99 eligible participants were recruited to the study. Of them, three patients were lost to followup in the experimental group due to home situations, one patient was admitted with psychiatric issues, and one had a change in BP medication, leading to a final total of 28 participants in the experimental group. The placebo group had three patients lost to followup due to home situations, one due to antihypertensive medication changes, and one had mild pruritus and dropped out after two attempts for participation, leading to a final total of 28 participants. The control group had four participants lost to followup due to 
TABLE 1: Homogeneity between groups.

\begin{tabular}{|c|c|c|c|c|c|}
\hline Categories & Aroma $(n=28)$ & Placebo $(n=28)$ & Control $(n=27)$ & $\chi^{2}$ or $F$ & $P$ \\
\hline & & Mean \pm SD & & & \\
\hline Age (yr.) & $52.5 \pm 4.6$ & $54.1 \pm 3.8$ & $53.3 \pm 4.6$ & 1.01 & .37 \\
\hline Height $(\mathrm{cm})$ & $157.1 \pm 6.9$ & $158.0 \pm 4.9$ & $158.4 \pm 3.8$ & 0.44 & .68 \\
\hline Weight (kg) & $61.8 \pm 7.4$ & $63.6 \pm 7.6$ & $64.2 \pm 7.0$ & 0.81 & .45 \\
\hline Age of menarche (yr.) & $15.6 \pm 1.68$ & $15.4 \pm 1.9$ & $15.6 \pm 1.8$ & 0.15 & .86 \\
\hline Age of first birth (yr.) & $26.1 \pm 4.3$ & $25.8 \pm 3.6$ & $26.6 \pm 4.5$ & 0.22 & .80 \\
\hline First SBP (mmHg) & $128.1 \pm 12.2$ & $127.5 \pm 14.4$ & $121.5 \pm 13.1$ & 2.06 & .13 \\
\hline First DBP (mmHg) & $79.7 \pm 9.5$ & $80.4 \pm 11.3$ & $80.1 \pm 10.6$ & 0.04 & .96 \\
\hline \multirow[t]{2}{*}{ First PR (bpm) } & $69.2 \pm 8.7$ & $71.0 \pm 12.4$ & $70.8 \pm 8.6$ & 0.26 & .77 \\
\hline & & $N(\%)$ & & & \\
\hline \multicolumn{6}{|l|}{ Marital status } \\
\hline Married & $26(92.9)$ & $26(92.9)$ & $22(81.5)$ & 3.44 & .30 \\
\hline Single & $2(7.1)$ & $2(7.1)$ & $5(18.5)$ & & \\
\hline \multicolumn{6}{|l|}{ Education } \\
\hline Middle school & $4(13.3)$ & $8(28.6)$ & $5(18.5)$ & 2.84 & .58 \\
\hline High school & $13(46.4)$ & $12(42.9)$ & $15(55.6)$ & & \\
\hline University & $11(39.3)$ & $8(28.6)$ & $7(25.9)$ & & \\
\hline \multicolumn{6}{|l|}{ Presence of menopause } \\
\hline Postmenopausal & $14(50.0)$ & $15(53.6)$ & $17(65.4)$ & 3.79 & .47 \\
\hline Intramenopausal & $8(28.6)$ & $10(35.7)$ & $4(15.4)$ & & \\
\hline No menopausal & $6(21.4)$ & $3(10.7)$ & $5(19.2)$ & & \\
\hline \multicolumn{6}{|l|}{ Drinking } \\
\hline No & $18(64.3)$ & $18(64.3)$ & $17(63.0)$ & 0.01 & .99 \\
\hline Yes & $10(35.7)$ & $10(35.7)$ & $10(37.0)$ & & \\
\hline \multicolumn{6}{|l|}{ Smoking } \\
\hline No & $27(96.4)$ & $28(100)$ & $25(92.6)$ & 2.17 & .34 \\
\hline Yes & $1(3.6)$ & $0(0.0)$ & $2(7.4)$ & & \\
\hline \multicolumn{6}{|l|}{ Exercise } \\
\hline No exercise & $5(17.9)$ & $8(28.6)$ & $7(25.9)$ & 2.13 & .71 \\
\hline Less than two times per week & $10(35.7)$ & $12(42.9)$ & $10(37.0)$ & & \\
\hline More than three times per week & $13(46.4)$ & $8(28.6)$ & $10(37.0)$ & & \\
\hline
\end{tabular}

DBP: diastolic blood pressure; PR: pulse rate; SBP: systolic blood pressure.

home situation, one patient who refused to participate due to inability to perform 24-hour ambulatory BP monitoring at work, and one patient who could not participate due to overseas travelling, leading to a final total of 27 participants. Therefore, there were 83 members in the study analyzed as final eligible study participants.

3.2. Homogeneity between the Groups. The general characteristics of the participants in the experimental, placebo, and control groups are shown in Table 1. No statistical differences were found between the groups with regards to marital status, level of education, presence of menopause, drinking, smoking, and exercise. There were also no significant differences in age, height, weight, age of menarche, age of first birth, baseline SBP, baseline DBP, and baseline pulse rate.

3.3. Effect of Aroma Massage on Home BP. The SBP and DBP were measured twice weekly for a total of eight measurements to evaluate the effect of aroma massage on participant home
BP (Table 2). The SBP measurements over four weeks did not show significant differences with time, and there was no interaction between the groups and time on repeated measures ANCOVA using initial SBP as a covariate. However, there were significant differences in repeated measurements of SBP depending on the group $(F=6.71, P=0.002)$. The post-hoc analysis with the Tukey method showed that the experimental group showed significant differences compared to the placebo group and the control group $(P<0.05)$.

The eight DBP measurements over four weeks did not show significant differences with time, and there was no interaction between the groups and time on repeated measures ANCOVA using initial DBP as a covariate. There was no difference in DBP measurements within the 3 groups.

3.4. Effect of Aroma Massage on Office BP. To determine the immediate effect of aroma massage, BP measurements were taken before the massage in the office followed by SBP measurement 10 minutes after the massage to compare with 
TABLE 2: Aroma massage on home BP.

\begin{tabular}{|c|c|c|c|c|c|}
\hline Time & Aroma $(n=28)$ & Placebo $(n=28)$ & Control. $(n=27)$ & $F$ & $P$ \\
\hline \multicolumn{6}{|c|}{ SBP } \\
\hline Baseline & $128.1 \pm 12.2$ & $127.5 \pm 14.4$ & $121.5 \pm 13.1$ & 2.06 & 0.13 \\
\hline \multicolumn{6}{|l|}{ 1st week } \\
\hline Tue. & $120.7 \pm 14.1$ & $121.2 \pm 12.4$ & $125.7 \pm 12.3$ & 1.24 & 0.29 \\
\hline Fri. & $118.5 \pm 13.8$ & $122.8 \pm 13.7$ & $123.3 \pm 11.0$ & 1.12 & 0.33 \\
\hline \multicolumn{6}{|l|}{ 2nd week } \\
\hline Tue. & $120.6 \pm 11.0$ & $121.6 \pm 12.3$ & $122.4 \pm 11.9$ & 0.16 & 0.85 \\
\hline Fri. & $117.9 \pm 10.1$ & $117.6 \pm 13.4$ & $121.7 \pm 11.3$ & 1.06 & 0.35 \\
\hline \multicolumn{6}{|l|}{ 3rd week } \\
\hline Tue. & $116.0 \pm 12.5$ & $118.6 \pm 12.7$ & $122.3 \pm 11.8$ & 1.77 & 0.18 \\
\hline Fri. & $114.7 \pm 12.6$ & $119.5 \pm 11.1$ & $121.9 \pm 12.0$ & 2.65 & 0.08 \\
\hline \multicolumn{6}{|l|}{4 th week } \\
\hline Tue. & $115.6 \pm 9.3$ & $121.6 \pm 12.1$ & $122.2 \pm 11.1$ & 3.21 & 0.05 \\
\hline Fri. & $113.3 \pm 11.0^{\mathrm{a}}$ & $121.8 \pm 11.3^{\mathrm{b}}$ & $120.9 \pm 12.1^{\mathrm{b}}$ & 4.66 & 0.01 \\
\hline \multicolumn{6}{|c|}{ Group: $F=6.71, P=0.002$; time: $F=0.46, P=0.86$; group $\times$ time: $F=1.68, P=0.07$} \\
\hline \multicolumn{6}{|c|}{ DBP } \\
\hline Baseline & $79.7 \pm 9.5$ & $80.4 \pm 11.3$ & $80.1 \pm 10.6$ & 0.04 & 0.96 \\
\hline \multicolumn{6}{|l|}{ 1st week } \\
\hline Tue. & $79.0 \pm 8.4$ & $77.8 \pm 9.1$ & $81.5 \pm 11.0$ & 1.06 & 0.96 \\
\hline Fri. & $78.8 \pm 9.9$ & $79.4 \pm 10.1$ & $81.5 \pm 11.0$ & 0.26 & 0.67 \\
\hline \multicolumn{6}{|l|}{ 2nd week } \\
\hline Tue. & $80.9 \pm 6.6$ & $78.7 \pm 6.6$ & $79.2 \pm 9.8$ & 0.52 & 0.36 \\
\hline Fri. & $79.9 \pm 8.3$ & $77.0 \pm 10.2$ & $79.6 \pm 9.1$ & 0.86 & 0.21 \\
\hline \multicolumn{6}{|l|}{ 3rd week } \\
\hline Tue. & $79.4 \pm 8.7$ & $76.4 \pm 11.5$ & $81.0 \pm 10.4$ & 1.45 & 0.13 \\
\hline Fri. & $77.9 \pm 9.5$ & $76.8 \pm 11.0$ & $80.1 \pm 7.6$ & 0.91 & 0.40 \\
\hline \multicolumn{6}{|l|}{ 4th week } \\
\hline Tue. & $77.5 \pm 9.1$ & $78.2 \pm 10.1$ & $79.4 \pm 8.6$ & 0.29 & 0.90 \\
\hline Fri. & $75.9 \pm 8.1$ & $77.4 \pm 10.5$ & $79.4 \pm 10.1$ & 0.89 & 0.66 \\
\hline
\end{tabular}

Values are expressed as mean \pm standard deviation.

Means for each group with different superscript (a, or b) indicate a significant difference (Tukey test; $P<0.05$ ).

BP: blood pressure; DBP: diastolic blood pressure; PR: pulse rate; SBP: systolic blood pressure.

the office BP. The postintervention office SBP, measured after each of the five massages, did not show significant differences between the two groups after the first massage. The aroma massage group showed significantly reduced SBP after the second $(t=-3.444, P=0.001)$, third $(t=-2.65, P=0.01)$, fourth $(t=-3.33, P=0.002)$, and fifth sessions $(t=-4.87$, $P<0.001)$. There was statistical significance in groups $\times$ time $(F=3.63, P=0.007)$ and the groups $(F=13.34, P=0.001)$, while there was not statistically significant in time.

Postintervention office DBP after the first session did not show significant differences between the two groups. However, there were significant differences between groups after the second $(t=-3.09, P=0.003)$, third $(t=-2.08$, $P=0.04)$, fourth $(t=-2.38, P=0.02)$, and fifth sessions $(t=-3.76, P<0.001)$ in office DBP. The results of repeated measures ANOVA showed significant difference with time $(F=3.84, P=0.005)$, groups $(F=8.46, P=0.005)$, and group $\times$ time $(F=2.79, P=0.03)$ (Table 3$)$.
3.5. Effect of Aroma Massage on 24-Hour Ambulatory BP. The 24-hour ambulatory BP was measured before and after the intervention for the experimental, placebo, and control groups. There were no significant differences between groups in 24-hour ambulatory daytime BP and 24-hour ambulatory nocturnal BP (Table 4).

3.6. Effect of Aroma Massage on Sleep Quality. There were significant differences between groups $(F=6.75, P=0.002)$ after intervention (Table 5$)$. There were significant differences in changes of sleep quality between groups $(F=9.32, P<$ 0.001). In a post-hoc analysis, the aroma massage group showed significant improvement in sleep quality compared with placebo and no-treatment groups (Tukey, $P<0.05$ ).

\section{Discussion}

This study investigated the effects of aroma massage on home BP, 24-hour ambulatory BP, and sleep quality in 
TABLE 3: Aroma massage on office BP.

\begin{tabular}{|c|c|c|c|c|}
\hline Time & Aroma $(n=28)$ & Placebo $(n=28)$ & $t$ & $P$ \\
\hline \multicolumn{5}{|c|}{ SBP } \\
\hline \multicolumn{5}{|c|}{ 1st session } \\
\hline Pre & $124.0 \pm 11.1$ & $125.8 \pm 15.7$ & -0.49 & 0.63 \\
\hline Post & $112.8 \pm 12.4$ & $118.9 \pm 15.2$ & -1.63 & 0.11 \\
\hline \multicolumn{5}{|c|}{ 2nd session } \\
\hline Pre & $119.0 \pm 11.7$ & $121.4 \pm 11.5$ & -0.78 & 0.44 \\
\hline Post & $108.5 \pm 10.8$ & $119.7 \pm 13.3$ & -3.44 & 0.001 \\
\hline \multicolumn{5}{|c|}{ 3rd session } \\
\hline Pre & $118.2 \pm 10.7$ & $122.3 \pm 13.1$ & -1.28 & 0.21 \\
\hline Post & $109.7 \pm 11.1$ & $118.6 \pm 13.9$ & -2.65 & 0.01 \\
\hline \multicolumn{5}{|c|}{ 4th session } \\
\hline Pre & $119.0 \pm 10.3$ & $125.1 \pm 15.5$ & -1.75 & 0.09 \\
\hline Post & $111.1 \pm 9.9$ & $122.9 \pm 15.9$ & -3.33 & 0.002 \\
\hline \multicolumn{5}{|c|}{ 5th session } \\
\hline Pre & $119.4 \pm 12.1$ & $126.6 \pm 15.7$ & -1.93 & 0.06 \\
\hline Post & $108.9 \pm 9.7$ & $125.0 \pm 14.6$ & -4.87 & $<0.001$ \\
\hline \multicolumn{5}{|c|}{$\begin{aligned} \text { Group: } F=13.34, P=0.001 ; \text { time: } F=2.15, P=0.08 & \\
& \text { group } \times \text { time: } F=3.63, P=0.007\end{aligned}$} \\
\hline \multicolumn{5}{|c|}{ DBP } \\
\hline \multicolumn{5}{|c|}{ 1st session } \\
\hline Pre & $74.3 \pm 7.7$ & $76.0 \pm 10.9$ & -0.67 & 0.51 \\
\hline Post & $71.8 \pm 8.4$ & $74.7 \pm 9.7$ & -1.19 & 0.24 \\
\hline \multicolumn{5}{|c|}{ 2nd session } \\
\hline Pre & $72.1 \pm 8.4$ & $74.3 \pm 8.0$ & -1.01 & 0.32 \\
\hline Post & $67.8 \pm 7.8$ & $74.8 \pm 9.3$ & -3.09 & 0.003 \\
\hline \multicolumn{5}{|c|}{ 3rd session } \\
\hline Pre & $72.7 \pm 7.7$ & $73.7 \pm 8.0$ & -0.50 & 0.62 \\
\hline Post & $70.2 \pm 7.8$ & $74.6 \pm 8.3$ & -2.08 & 0.04 \\
\hline \multicolumn{5}{|c|}{ 4th session } \\
\hline Pre & $73.1 \pm 6.8$ & $76.0 \pm 9.8$ & -1.27 & 0.21 \\
\hline Post & $72.0 \pm 6.7$ & $77.4 \pm 10.1$ & -2.38 & 0.02 \\
\hline \multicolumn{5}{|c|}{ 5th session } \\
\hline Pre & $72.6 \pm 8.3$ & $76.0 \pm 9.9$ & -1.38 & 0.18 \\
\hline Post & $69.5 \pm 6.4$ & $78.1 \pm 10.3$ & -3.76 & $<0.001$ \\
\hline
\end{tabular}

Values are expressed as mean \pm standard deviation.

BP: blood pressure; DBP: diastolic blood pressure; SBP: systolic blood pressure.

middle-aged women with hypertension. The results show that aroma massage significantly reduced home SBP compared with the placebo and no-treatment groups.

The aroma massage group showed an approximate reduction of $15 \mathrm{mmHg}$ for home SBP, while the placebo group showed an approximate reduction of $6 \mathrm{mmHg}$. One previous study showed a reduction of $4 \mathrm{mmHg}$ in SBP using posterior neck massage three times a week for six weeks with lavender oil [15]. Comparing with these results, the effect observed with once-weekly overall body massage in this study shows that it is very effective in lowering BP. The oils used in this study (a blend of lavender, ylang-ylang, marjoram, and
TABLE 4: Aroma massage on 24-hour ambulatory BP.

\begin{tabular}{lccccc}
\hline Time & $\begin{array}{c}\text { Aroma } \\
(n=28)\end{array}$ & $\begin{array}{c}\text { Placebo } \\
(n=28)\end{array}$ & $\begin{array}{c}\text { Control } \\
(n=27)\end{array}$ & $F$ & $P$ \\
\hline \multicolumn{7}{c}{ SBP } \\
\hline $\begin{array}{c}\text { Daytime } \\
\text { Pre }\end{array}$ & $134.8 \pm 12.0$ & $136.2 \pm 13.5$ & $128.5 \pm 12.5$ & 2.88 & .06 \\
$\quad \begin{array}{c}\text { Post } \\
\text { Night time }\end{array}$ & $130.3 \pm 11.7$ & $128.4 \pm 12.2$ & $133.9 \pm 12.9$ & 1.37 & .26 \\
$\quad$ Pre & $122.2 \pm 15.8$ & $124.9 \pm 13.9$ & $119.1 \pm 16.4$ & 0.98 & .38 \\
$\quad$ Post & $117.5 \pm 15.6$ & $119.0 \pm 9.9$ & $122.9 \pm 18.1$ & 0.93 & .40 \\
\hline & & DBP & & & \\
\hline Daytime & & & & & \\
$\quad$ Pre & $82.7 \pm 8.6$ & $83.6 \pm 8.1$ & $81.6 \pm 9.4$ & 0.37 & .69 \\
$\quad$ Post & $81.4 \pm 8.3$ & $80.0 \pm 8.1$ & $83.4 \pm 8.9$ & 1.07 & .35 \\
Night time & & & & & \\
$\quad$ Pre & $74.8 \pm 9.9$ & $76.4 \pm 8.9$ & $74.4 \pm 11.1$ & 0.30 & .74 \\
$\quad$ Post & $73.6 \pm 10.5$ & $71.7 \pm 6.8$ & $74.6 \pm 11.0$ & 0.59 & .55 \\
\hline
\end{tabular}

Values are expressed as mean \pm standard deviation.

BP: blood pressure; DBP: diastolic blood pressure; SBP: systolic blood pressure.

TABLE 5: Aroma massage on sleep quality.

\begin{tabular}{lccccc}
\hline Time & $\begin{array}{c}\text { Aroma } \\
(n=28)\end{array}$ & $\begin{array}{c}\text { Placebo } \\
(n=28)\end{array}$ & $\begin{array}{c}\text { Control. } \\
(n=27)\end{array}$ & $F$ & $P$ \\
\hline Pre & $51.0 \pm 12.6$ & $48.1 \pm 15.8$ & $55.6 \pm 10.5$ & 2.24 & 0.11 \\
Post & $58.1 \pm 11.3^{\mathrm{a}}$ & $46.5 \pm 13.8^{\mathrm{b}}$ & $50.3 \pm 10.9^{\mathrm{b}}$ & 6.75 & 0.002 \\
Difference & $7.1 \pm 12.6^{\mathrm{a}}$ & $-1.6 \pm 9.6^{\mathrm{b}}$ & $-5.3 \pm 10.6^{\mathrm{b}}$ & 9.32 & $<0.001$ \\
\hline
\end{tabular}

Values are expressed as mean \pm standard deviation.

Means for each group with different superscript ( $a, b$, or $c)$ indicate a significant difference (Tukey test; $P<0.05$ ).

Neroli) are thought to have produced synergistic effects in lowering BP compared with a simple lavender oil [13]. In addition, previous research using inhalation therapy using lavender, ylang-ylang, and bergamot showed a decrease of $19 / 10 \mathrm{mmHg}$ in BP and showed synergistic effects in lowering BP compared to this study [14].

The aroma massage may reduce $\mathrm{BP}$ by inducing the physical relaxation and decreasing activation of sympathetic nervous system. In this study, the placebo group with artificial fragrance also showed decreased $\mathrm{BP}$ with massage. This is thought to be due to the effect of massage rather than the artificial fragrance.

In particular, the office SBP decreased by $12 \mathrm{mmHg}$ after the first massage and $11 \mathrm{mmHg}$ after the fifth massage in the aroma massage group. This shows the acute effect of aroma massage for immediate decreasing of BP. The BP decrease after the first massage in the placebo group was approximately $7 \mathrm{mmHg}$. However, it did not show substantial reduction as massage sessions progressed, pointing to a temporary effect of massage with artificial fragrance.

The home DBP decreased by approximately $4 \mathrm{mmHg}$ in the experimental group, $3 \mathrm{mmHg}$ in the placebo group, and $1 \mathrm{mmHg}$ in the control group, showing no difference among the groups. The office DBP before and after massage showed 
a reduction of approximately $5 \mathrm{mmHg}$ in the experimental group as opposed to an elevation of $2 \mathrm{mmHg}$ in the placebo group. The effect of aroma massage in reducing DBP is limited, although there appears to be some immediate reducing effect of aroma massage for office DBP.

There was no difference in 24-hour ambulatory BP between groups. A once-weekly intervention of aroma massage and cream application does not appear to promote positive 24-hour ambulatory BP. Therefore, further research on the frequency of massage and the active ingredients and types of oils used is necessary.

In terms of sleep quality, the aroma massage group showed an improvement, while the placebo and no-treatment groups failed to do so. This result appears to be due to the effect of the essential oil used in this study on the autonomic nervous system to increase the quality of sleep [7, 9]. These results are in line with those of a study by Jung and Jeon [16]. They used a mixture of lavender, bergamot, and clary sage in a $3: 2: 1$ ratio applied to hemiplegic patients on the arm, hand, and the lower neck through aroma massage and showed reduction of sleep disturbance score. The results of this study are consistent with the other study of middle-aged women using a lavender essential oil necklace and one to two drops of lavender essential oil on the pillow before sleep (instead of the necklace) [17], which showed improvement in sleep quality.

Our study did not take into account the preference of participants for aroma fragrance. Although most participants reported a good feeling toward the aroma, some reported preference for another fragrance. Thus, the psychological and physical responses may vary depending on the fragrance preference of the subject receiving the aromatherapy.

In conclusion, aroma massage was effective in reducing home SBP and immediate office BP before and after the intervention as well as increasing sleep quality in middleaged women with hypertension. Further research comparing the effects of various and convenient aromatherapy methods including bathing, topical application, and foot washing is needed.

\section{Conflict of Interests}

The authors declare no conflict of interests.

\section{Acknowledgment}

This work was supported by the National Research Foundation of Korea (NRF) Grant funded by the Korean government MEST (no. 2008-0061658). M. S. Lee was supported by Korea Institute of Oriental Medicine (K12130).

\section{References}

[1] World Health Organization, Chronic Diseases, World Health Organization, Geneva, Switzerland, 2012.

[2] National Health Statistics, "National health nutrition survey quaternary period secondary source," Approval Number Statistics 11702, The Ministry of Health and Welfare, 2008.
[3] B. H. Lee, Manual of Hypertension, Deahanuihakseojeok, Seoul, Korea, 2009.

[4] P. Bansil, E. V. Kuklina, R. K. Merritt, and P. W. Yoon, "Associations between sleep disorders, sleep duration, quality of sleep, and hypertension: results from the National Health and Nutrition Examination Survey, 2005 to 2008," Journal of Clinical Hypertension, vol. 13, no. 10, pp. 739-743, 2011.

[5] D. D. Smeltzer, B. G. Bare, J. L. Hinkle, and K. H. Cheever, Brunner \& Suddarth's Textbook of Medical-Surgical Nursing, Wolsters Kluwer/Lippincott Williams \& Wilkins, Philadelphia, $\mathrm{Pa}, \mathrm{USA}, 2010$.

[6] S. Battaglia, The Complete Guide to Aromatherapy, The International Centre of Holistic Aromatherapy, Brisbane, Australia, 2003.

[7] B. J. Ha, Aromatherapy, Soo Moon Sa, Seoul, Korea, 2006.

[8] P. Daniel, P. Shirley, and P. Len, Aromatherphy for Healt Professionals, Churchill Livingstone, London, UK, 2001.

[9] S. H. Han, M. H. Hur, J. H. Park, E. J. Lee, and M. K. Park, Women's Health and Aromatherapy, . Hyun Moon Sa, Seoul, Korea, 2002.

[10] J. F. Owens and K. A. Matthews, "Sleep disturbance in healthy middle-aged women," Maturitas, vol. 30, no. 1, pp. 41-50, 1998.

[11] P. Vara, "The climacterium from the gynaecologist/s point of view," Acta Obstetricia et Gynecologica Scandinavica. Supplement, vol. 49, supplement 1, pp. 43-55, 1970.

[12] M. H. Hur, H. Oh, M. S. Lee, C. Kim, A. N. Choi, and G. R. Shin, "Effects of aromatherapy massage on blood pressure and lipid profile in Korean climacteric women," International Journal of Neuroscience, vol. 117, no. 9, pp. 1281-1287, 2007.

[13] Y. J. Jung, Effects of Aromatherapy on Blood Pressure, Heart Rate Variability, and Serum Catecholamines in the Pre-Hypertension Middle Aged Women, Department of Nursing, Catholic University, Seoul, Korea, 2007.

[14] J. H. Hwang, "The effects of the inhalation method using essential oils on blood pressure and stress responses of clients with essential hypertension," Journal of Korean Academy of Nursing, vol. 36, no. 7, pp. 1123-1134, 2006.

[15] Y. K. Choi and S. Lee, "The effect of posterior neck massage using maychang oil on the blood pressure and components in elderly women who have hypertension," Journal of Korean Beauty Society, vol. 17, no. 2, pp. 211-217, 2011.

[16] H. M. Jung and Y. S. Jeon, "Effects of the aroma massage on shoulder pain, depression, sleep disturbance in hemiparesis patients," The Korean Journal of Rehabilitation Nursing, vol. 7, no. 2, pp. 231-237, 2004.

[17] G. H. Lee, K. M. Park, and M. K. Ryu, "The effect of aromatherapy with lavender essential oil on sleep disturbance and depression on middle-aged women," Journal of the Korean Society of Maternal and Child Health, vol. 6, no. 1, pp. 23-37, 2002. 


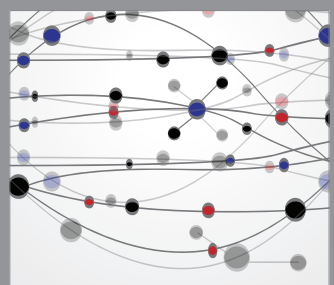

The Scientific World Journal
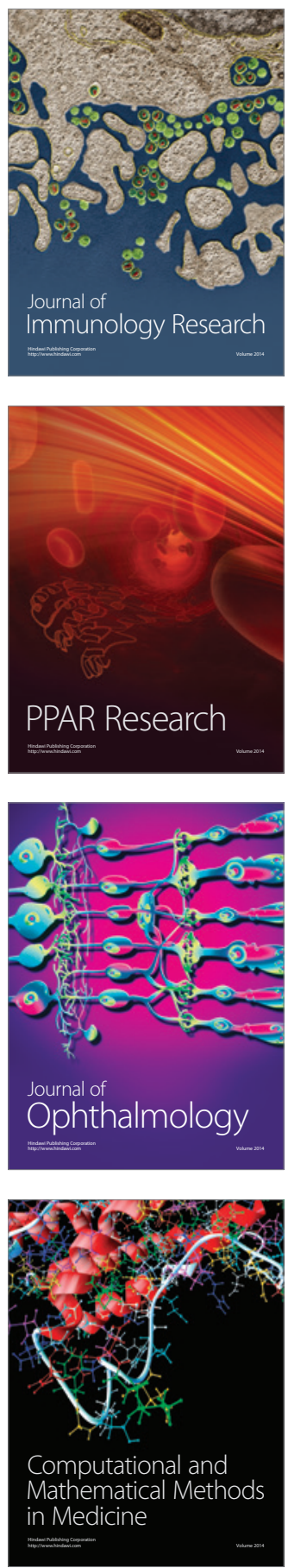

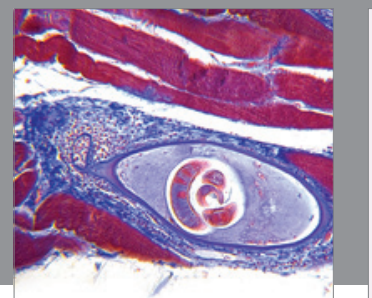

Gastroenterology

Research and Practice
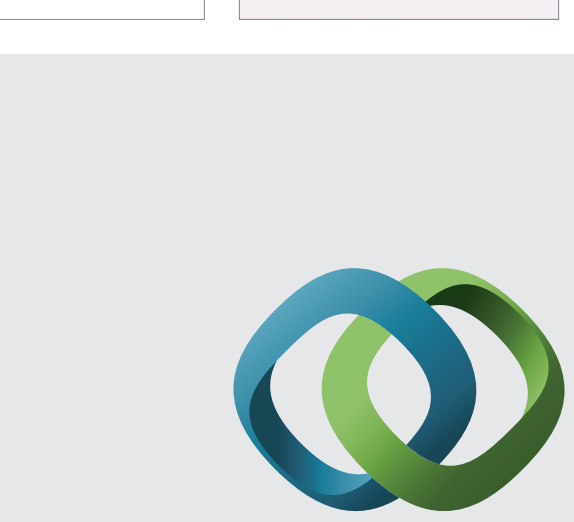

\section{Hindawi}

Submit your manuscripts at

http://www.hindawi.com
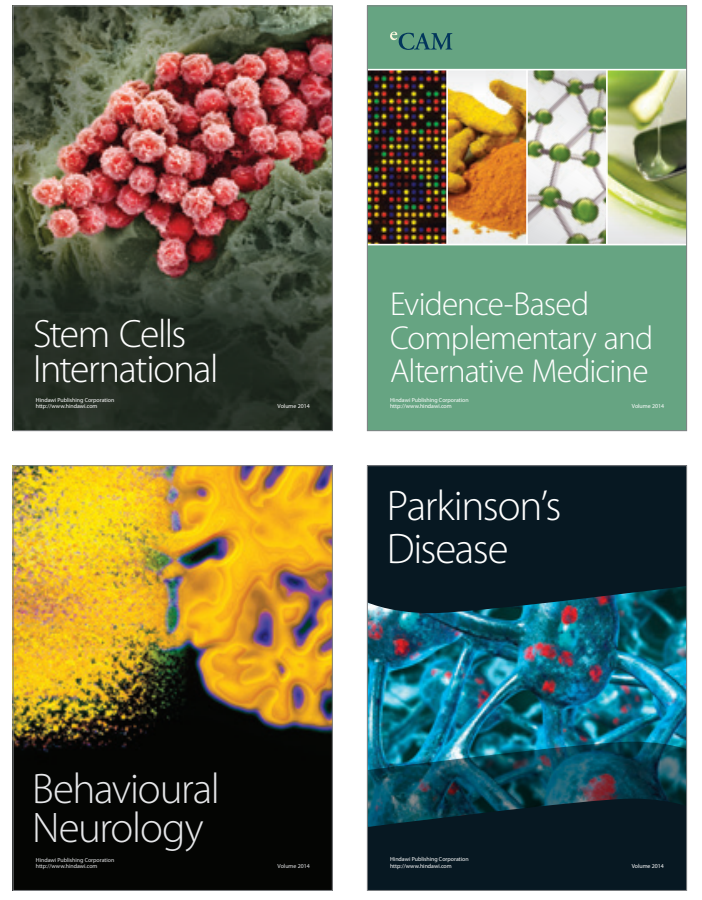
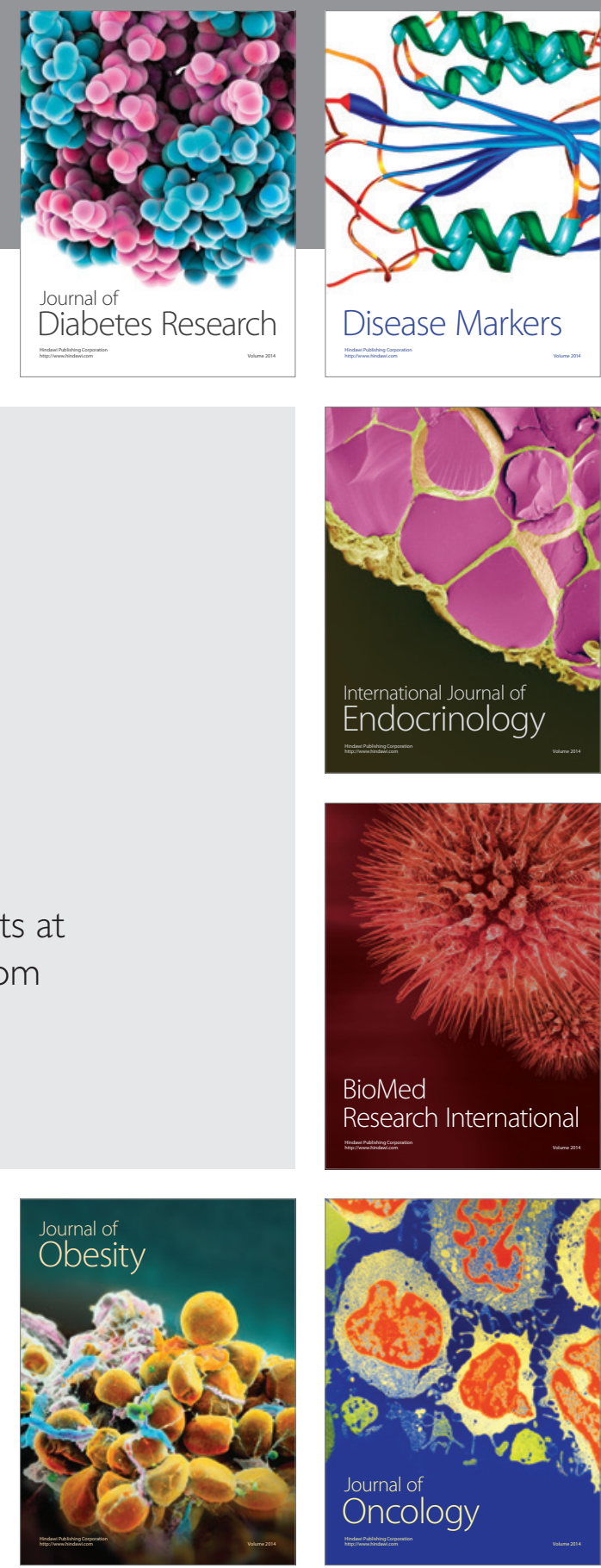

Disease Markers
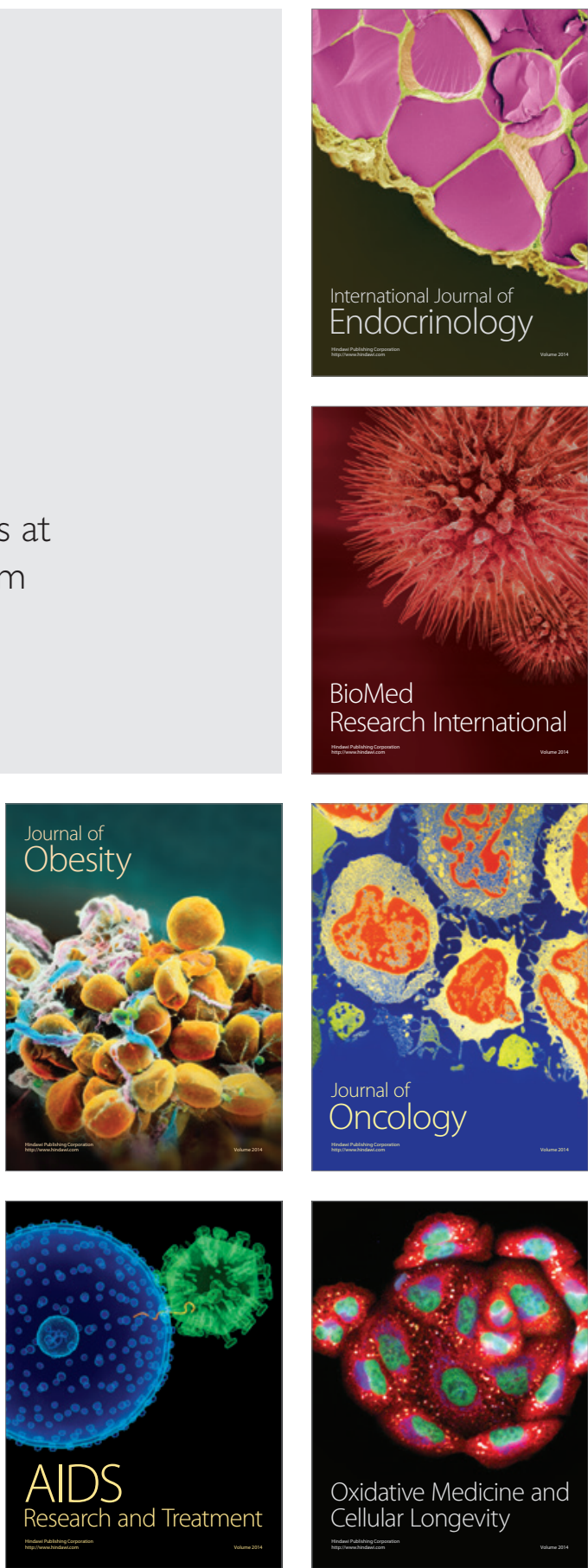